\section{(6) OPEN ACCESS}

\title{
Liver transplantation for hepatocellular carcinoma beyond the Milan criteria
}

\author{
Xiao Xu, ${ }^{1,2}$ Di Lu, ${ }^{1}$ Qi Ling, ${ }^{1}$ Xuyong Wei, ${ }^{1}$ Jian Wu, ${ }^{1}$ Lin Zhou, ${ }^{1}$ Sheng Yan, ${ }^{1}$ \\ Liming Wu, ${ }^{1}$ Lei Geng, ${ }^{1}$ Qinghong Ke, ${ }^{1}$ Feng Gao, ${ }^{1}$ Zhenhua Tu, ${ }^{1}$ Weilin Wang, ${ }^{1}$ \\ Min Zhang, ${ }^{1}$ Yan Shen, ${ }^{1}$ Haiyang Xie, ${ }^{1}$ Wenshi Jiang, ${ }^{3}$ Haibo Wang, ${ }^{3}$ \\ Shusen Zheng ${ }^{1,2}$
}

\begin{abstract}
- Additional material is published online only. To view please visit the journal online (http://dx.doi.org/10.1136/ gutjnl-2014-308513)

${ }^{1}$ Division of Hepatobiliary and Pancreatic Surgery, Department of Surgery, First Affiliated Hospital, Zhejiang University School of Medicine, Hangzhou, China

${ }^{2}$ Collaborative Innovation Center for Diagnosis and Treatment of Infectious Diseases, Hangzhou, China ${ }^{3}$ China Liver Transplant Registry, Shenzhen, China
\end{abstract}

\section{Correspondence to} Professor Shusen Zheng Division of Hepatobiliary and Pancreatic Surgery, Department of Surgery, First Affiliated Hospital, Zhejiang University School of Medicine, 79 Qingchun Road, Hangzhou 310003, China; zyzss@zju.edu.cn

Received 24 September 2014 Revised 3 March 2015 Accepted 4 March 2015 Published Online First 24 March 2015

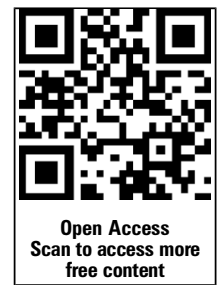

CrossMark

To cite: Xu X, Lu D, Ling Q, et al. Gut 2016;65:

1035-1041.

\section{ABSTRACT}

Objective Liver transplantation is an optimal radical therapy for selected patients with hepatocellular carcinoma. The stringent organ allocation system driven by the Milan criteria has been challenged by alternative sets of expanded criteria. Careful analysis is needed to prove that the Milan criteria can be expanded safely and effectively.

Design This study collectively reviewed 6012 patients of hepatocellular carcinoma from the China Liver Transplant Registry. Expanded criteria were evaluated to characterise an optimised expansion with acceptable outcomes beyond the Milan criteria.

Results Compared with the Milan criteria, Valencia, University of California, San Francisco, University Clinic of Navarra and Hangzhou criteria provided an expansion of $12.4 \%, 16.3 \%, 19.6 \%$, and $51.5 \%$, respectively. The post-transplant survivals of patients fulfilling the expanded criteria were comparable to that of the Milan criteria. The analysis of net reclassification improvement and area under the receiver operating characteristic curves showed an excellent efficiency in recurrence prediction for the expanded criteria compared with the Milan criteria. In patients exceeding Milan but fulfilling the Hangzhou criteria ( $N=1352$ ), $\alpha$-fetoprotein (AFP) $>100 \mathrm{ng} / \mathrm{mL}$ and tumour burden $>8 \mathrm{~cm}$ were the only two independent prognostic factors $(p<0.001)$.

Accordingly, the Hangzhou criteria were stratified as type A (tumour burden $\leq 8 \mathrm{~cm}$, or tumour burden $>8 \mathrm{~cm}$ but AFP $\leq 100 \mathrm{ng} / \mathrm{mL}$ ) and type $B$ (tumour burden $>8 \mathrm{~cm}$ but AFP between 100 and $400 \mathrm{ng} / \mathrm{mL}$ ). Type A showed significantly higher 5 -year tumour-free survival rates compared with type $B(p<0.001)$.

Conclusions The Milan criteria can be expanded safely and effectively. The prognostic stratification system based on the Hangzhou criteria serves as a hierarchy of transplant candidates for hepatocellular carcinoma.

\section{INTRODUCTION}

Hepatocellular carcinoma (HCC) has been increasingly prevalent throughout the world, with the seventh highest cancer rate and the third highest cancer mortality. ${ }^{1}{ }^{2}$ China has the heaviest HCC burden worldwide, accounting for $55 \%$ of all newly diagnosed HCC cases and around $45 \%$ of deaths from HCC in the world. ${ }^{3}{ }^{4}$ Liver transplantation is regarded as an optimal radical therapy for selected patients with HCC. Data from liver

\section{Significance of this study}

What is already known on this subject?

- Liver transplantation is an optimal radical therapy for selected patients with hepatocellular carcinoma (HCC).

- It is well known that the Milan criteria are the golden candidate selection criteria that ensure excellent post-transplant survival for patients with $\mathrm{HCC}$.

- The stringent organ allocation system driven by the Milan criteria has been challenged by alternative sets of expanded criteria.

\section{What are the new findings?}

- This was a pioneering study comparing the efficiency and safety of different criteria in the candidate selection for liver transplantation based on the largest HBV-related HCC cohort.

- The Valencia, University of California, San Francisco, University Clinic of Navarra and Hangzhou criteria provided expansions to the Milan criteria without significant impairment in the post-transplant survival. Among the four sets of criteria, the Hangzhou criteria had the greatest expansion as well as excellent prognostic-predicting capacity.

- Type A of Hangzhou criteria had significantly better survival than type $B$, and should have the priority for liver transplantation.

How might it impact on clinical practice in the foreseeable future?

- The Milan criteria can be safely and effectively expanded, and the prognostic stratification system can be used in candidate selection for liver transplantation in patients with HCC.

transplant registries showed that China has the greatest HCC candidate list and almost half of liver transplants were performed for HCC in the past decades.

It is well known that the Milan criteria are the golden candidate selection criteria that ensure excellent post-transplant survival for patients with HCC. ${ }^{5}{ }^{6}$ However, growing experience of liver transplantation for HCC raised concerns about the 
Milan criteria as being too restrictive and far from satisfying the increasing candidate list, particularly in China. ${ }^{7}$ Therefore, careful expansion to the Milan criteria has been proposed, including the University of California, San Francisco (UCSF), ${ }^{8}$ University Clinic of Navarra (CUN), ${ }^{9}$ Valencia $^{10}$ and Hangzhou criteria $^{11}$ (see online supplemental table S1). These alternative sets of criteria imply that the Milan criteria can be expanded. However, there are debates on whether such expansions are appropriate and which criteria to use. ${ }^{12}$

In this study, we collectively reviewed 6012 patients with HCC undergoing liver transplantation from the China Liver Transplant Registry (CLTR). It is the third largest liver transplant database in the world. Based on this large HCC patient cohort undergoing transplantation, the present study aimed to characterise an ideal candidate selecting system beyond the Milan criteria.

\section{PATIENTS AND METHODS \\ Patients and data}

The patient cohorts derived from the ongoing CLTR database. Until 31 December 2012, the CLTR covered a total of 23805 cases of liver transplantation. The subject selection process is depicted in figure 1 . This study excluded patients with incomplete follow-up, missing essential data for analysis (tumour size, number, differentiation grade, $\alpha$-fetoprotein (AFP)) or vascular invasion according to radiological criteria, and had 6554 patients available for analysis. Finally, after excluding those patients with perioperative mortality ( $<30$ days, $\mathrm{N}=542)$, altogether 6012 patients were studied. The major causes of deaths included haemorrhage, infection, graft failure and multiple organ dysfunction syndromes. All of them were histologically confirmed by postoperative pathological examination in the participating centres. The donor-to-recipient arrangements all conformed to the principle of $\mathrm{ABO}$ compatibility. Patients should be excluded for liver transplantation as long as extrahepatic metastasis and vascular invasion were detected before the operation, except for some transplants performed on patients with predetected vascular invasion in the 1990s and early 20th century. Among the 6012 patients, 5393 were men and 619 women. The age of the patients ranged from 18 to 74 years (mean, 50.3 \pm 8.7 years). Most of the patients $(91.2 \%, \mathrm{~N}=5483$ ) were hepatitis B surface antigen (HBsAg) positive. Liver cirrhosis was present in $86.2 \%(\mathrm{~N}=5185)$ of the patients. There were $794(13.2 \%)$ patients receiving salvage liver transplantation due to tumour recurrence after hepatectomy. Before transplantation, 1813 patients $(30.2 \%)$ received transcatheter arterial chemoembolisation and 270 patients (4.5\%) underwent radiofrequency ablation. The radiological information was acquired from the latest CT or MRI examination before liver transplantation.

Catalogued data included demographics, preoperative serum AFP level, morphological features (cirrhosis, tumour size, number of nodules), the model for end-stage liver diseases score, HBsAg positivity, tumour, node, metastases (TNM) grade (Union for International Cancer Control), adjuvant tumour therapy, donor origin, tumour differentiation grades (based on Edmondson-Steiner grading ${ }^{13}$ ) and vascular invasion (according to post-transplant pathology), tumour recurrence and patient survival. For tumour morphological features (according to the imaging), CLTR compares the pathological results and imaging findings to ensure the reliability of data. If obvious differences were present in a certain case, it should not be enrolled for analysis. This study randomly selected 200 patients from the whole cohort for the comparisons of tumour size and number between imaging and post-transplant pathology, which is shown in online supplemental figure S1.

The radiological diagnostic modality was mainly based on CT and MRI. The pretransplant imaging protocol included US every week, CT or MRI every four weeks since a patient's first appearance in the waiting list. Those who progressed into late stage (eg, extrahepatic metastasis and vascular invasion) during the waiting time were excluded from the candidate list.

\section{Statistical analysis}

Endpoints for the current analysis were patient death or tumour recurrence. Overall and tumour-free survival rates were calculated using the Kaplan-Meier method. Log-rank test was used to perform the univariate analysis, and Cox proportional hazard regression models were used for multivariate analysis. Those variables, which were found to be significant in univariate analysis, were further enrolled in the multivariate analysis. Net reclassification improvement (NRI) was estimated to compare the efficiency of risk reclassification for tumour recurrence. ${ }^{14}$ The area under the receiver operating characteristic curve (AUROC) value was calculated for the discriminatory ability of each set of criteria. ${ }^{15}$ A multivariate Cox model was built comprising all the five criteria as covariates. By removing a certain set of criteria individually from the full model, its independent contribution was evaluated in regards to the changes in likelihood ratio test
Figure 1 Flow chart of patient selection procedures. HCC, hepatocellular carcinoma; LT, liver transplant.

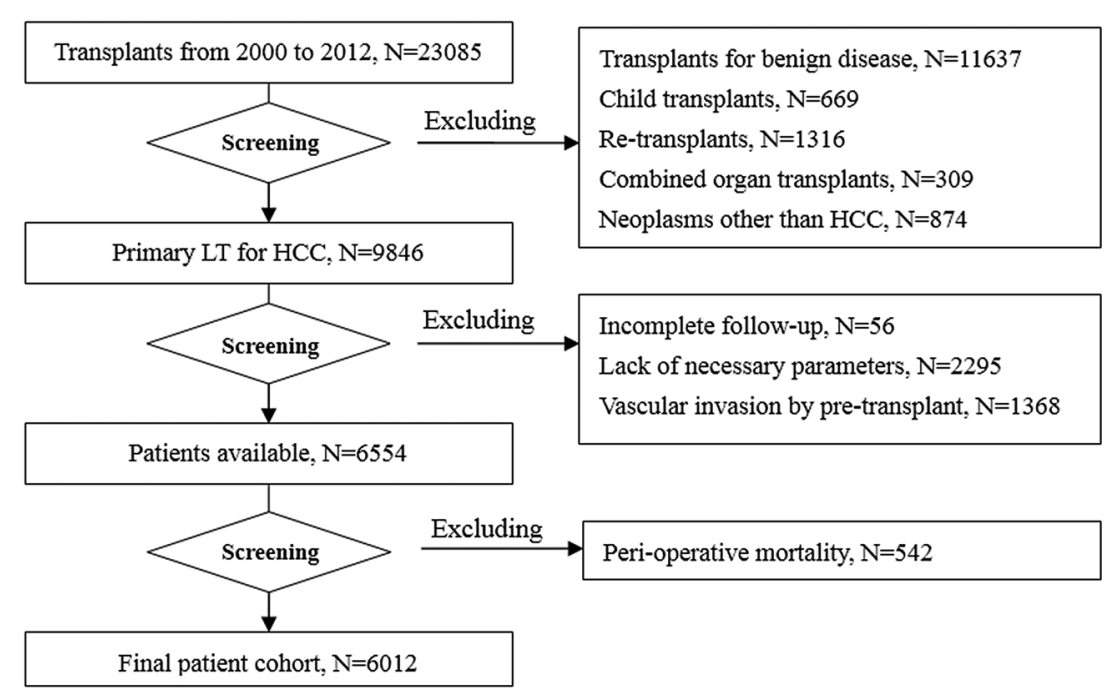


(LRT) $\chi^{2}$ and Akaike information criterion (AIC) value. ${ }^{16} 17$ Higher LRT $\chi^{2}$ indicates higher homogeneity in the prognosis for patients in the same category. ${ }^{18}$ When the AIC value is lower, the model is more accurate and informative. ${ }^{19} \mathrm{~A} p$ value $<0.05$ was considered statistically significant. The statistical measurements were performed using the SAS, V.8.0 (SAS Institute, Cary, North Carolina, USA) software program.

\section{RESULTS}

\section{Evaluation of the Milan criteria}

The median follow-up length is 31.9 months (ranging from 3.0 to 154.4 months). In the 6012 patients, $43.7 \%(\mathrm{~N}=2626)$ fulfilled the Milan criteria. The 1-year, 3-year, 5-year and 10-year tumour-free survival for patients fulfilling and exceeding the Milan criteria were $87.3 \%, 77.0 \%, 73.0 \%$ and $53.0 \%$ vs $67.7 \%, 46.8 \%, 39.5 \%$ and $24.3 \%(\mathrm{p}<0.001$, figure 2 and online supplemental table S2). In the 3386 patients exceeding the Milan criteria, 2255 patients $(66.6 \%)$ did not have tumour recurrence during the 5 -year follow-up.

\section{Expansion to the Milan criteria}

Compared to the Milan criteria, the Valencia, UCSF, CUN and Hangzhou criteria provided an expansion of $12.4 \%(\mathrm{~N}=325)$, $16.3 \%(\mathrm{~N}=429), 19.6 \% \quad(\mathrm{~N}=516)$ and $51.5 \% \quad(\mathrm{~N}=1352)$, respectively (figure $3 \mathrm{~A}$ ). The tumour-free survival rates of the patients fulfilling the expanded criteria were comparable to those of the Milan criteria $(\mathrm{p}>0.05)$. And similar to the Milan criteria, patients fulfilling the Valencia, UCSF, CUN and Hangzhou criteria had significantly better overall and tumourfree survival compared with those exceeding the corresponding criteria ( $\mathrm{p}<0.001$, figure 2 and online supplementary table S2). For the patient cohort including those with perioperative mortality, the results are shown in online supplementary figure S2 and table S3.

\section{Prognostic power of different set of criteria}

Tables were constructed for the net reclassification of patients according to different criteria (see online supplemental table S4). As compared with the Milan criteria, all the four expanded criteria improved the efficiency of risk reclassification in regards to the 5 -year tumour recurrence $(\mathrm{p}<0.01)$.

Plots for the time-dependent NRI value are depicted in figure $3 \mathrm{~B}$. During the entire course of 5 -year follow-up, the Valencia, UCSF, CUN and Hangzhou criteria all maintained a positive improvement compared with the Milan criteria. Among the four expanded criteria, the improvement referring to the Hangzhou criteria was marked in the first two years following liver transplantation.

The time-dependent AUROC curves are depicted in figure 3C. During the entire course of 5-year follow-up, the Valencia, UCSF, CUN and Hangzhou criteria all maintained higher AUROC values than the Milan criteria. The Hangzhou criteria were distinguished among these criteria in the first two years after transplantation.

According to the model built comprising all the five set of criteria, removing the Hangzhou criteria resulted in the greatest loss in the LRT $\chi^{2}$, as well as the greatest increase in the AIC value (table 1). It indicated that the Hangzhou criteria made the largest contribution to the full model regarding the prognostic ability.

\section{Exceeding the Milan criteria}

In patients exceeding the Milan criteria $(\mathrm{N}=3386)$, univariate analysis identified younger age ( $\leq 50$ years), liver cirrhosis, poor differentiation, tumour TNM stage (III or worse), tumour burden (the largest diameter of single tumour or the cumulative tumour diameters of multiple tumours, $>8 \mathrm{~cm}$ ), vascular invasion, elevated serum AFP ( $>400 \mathrm{ng} / \mathrm{mL})$ and transplants before 2005 as the risk factors for tumour recurrence (see online supplementary table S5). If taken as a single parameter and entered into multifactor Cox regression (relevant variables being excluded), 'exceeding the Hangzhou criteria' turned out to be an independent risk factor for tumour recurrence in patients exceeding the Milan criteria (table 2). The 1-year, 3-year, 5-year and 10-year overall survival rates for the patients exceeding the Milan criteria but fulfilling the Hangzhou criteria $(\mathrm{N}=1352)$ and those exceeding the Hangzhou criteria $(\mathrm{N}=2034)$ were $89.5 \%, 70.8 \%, 62.4 \%$ and $52.9 \%$ vs $73.0 \%, 42.9 \%, 32.8 \%$ and $22.3 \%$, respectively $(\mathrm{p}<0.001)$. And the 1 -year, 3 -year, 5 -year and 10-year tumour-free survival rates were $81.6 \%$, $64.3 \%, 56.5 \%$ and $37.2 \%$ vs $58.2 \%, 35.1 \%, 28.2 \%$ and $16.3 \%$, respectively $(\mathrm{p}<0.001$, figure 4$)$.

\section{Exceeding the Milan but fulfilling the Hangzhou criteria}

In those patients exceeding the Milan criteria but fulfilling the Hangzhou criteria $(\mathrm{N}=1352)$, both the univariate and multivariate analyses showed that AFP $>100 \mathrm{ng} / \mathrm{dL}$ and tumour size $>8 \mathrm{~cm}$ were the only two independent risk factors for tumour recurrence (table 3 and online supplemental table S6). These patients were accordingly divided into subsets I (AFP $\leq 100 \mathrm{ng}$ / $\mathrm{mL}$ or tumour burden $\leq 8 \mathrm{~cm}, \mathrm{~N}=1201$ ) and II (AFP $>100 \mathrm{ng} /$ $\mathrm{mL}$ and tumour burden $>8 \mathrm{~cm}, \mathrm{~N}=151)$. The 1 -year, 3 -year and 5 -year tumour-free survival rates for patients in subsets I and II were $83.1 \%, 67.0 \%$ and $59.8 \%$ vs $71.3 \%, 47.8 \%$ and $38.8 \%$, respectively $(\mathrm{p}<0.001$, figure $5 \mathrm{~A})$. Both subsets exhibited significantly greater prognosis compared with those patients exceeding the Hangzhou criteria (see online supplemental table S7).

\section{The stratification of the Hangzhou criteria: A and B}

This study then stratified the Hangzhou criteria as type A (tumour burden $\leq 8 \mathrm{~cm}$ regardless of AFP and differentiation, or tumour burden $>8 \mathrm{~cm}$ but AFP $\leq 100 \mathrm{ng} / \mathrm{mL}$ and well-moderate differentiation, $\mathrm{N}=3827$ ) and type $\mathrm{B}$ (tumour burden $>8 \mathrm{~cm}$ but AFP between 100 and $400 \mathrm{ng} / \mathrm{mL}$ and well-moderate differentiation, $\mathrm{N}=151$ ), as illustrated in online supplemental figure $\mathrm{S} 3$.

The 1-year, 3-year and 5-year tumour-free survival rates were $86.1 \%, 74.4 \%$ and $69.5 \%$ vs $71.3 \%, 47.8 \%$ and $38.8 \%$ for types A and B, respectively $(\mathrm{p}<0.001)$. Patients of both types had significantly improved prognosis compared with those exceeding the Hangzhou criteria (figure 5B).

\section{DISCUSSION}

The Milan criteria represent a milestone in the development of liver transplantation. The implementation of this set of criteria in the United Network for Organ Sharing system proved it successful in the assignment of listing priority for patients with HCC. ${ }^{20}$ However, concerns remained that the restrictive prerequisites might discard a substantial number of patients who could otherwise have done well after transplantation. In particular in China, around $40 \%$ of donor livers are allocated to HCC recipients. If strictly adhered to the Milan criteria, only $43.8 \%$ of patients in this study would have the opportunity of transplantation. Meanwhile, in those patients exceeding the Milan criteria, there were still two-thirds of patients who did not have tumour recurrence during the 5-year follow-up. Current organ allocation policies based on the Milan criteria do not adapt to the development of liver transplantation. 


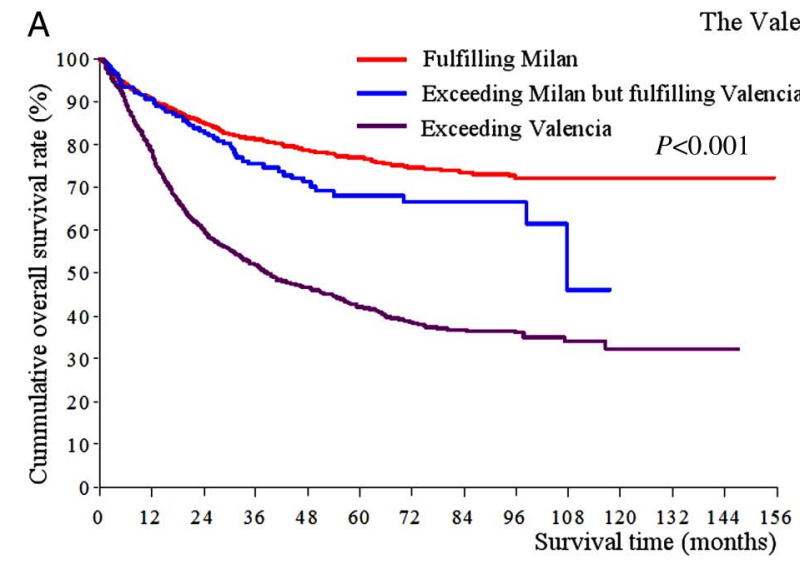

The Valencia Criteria
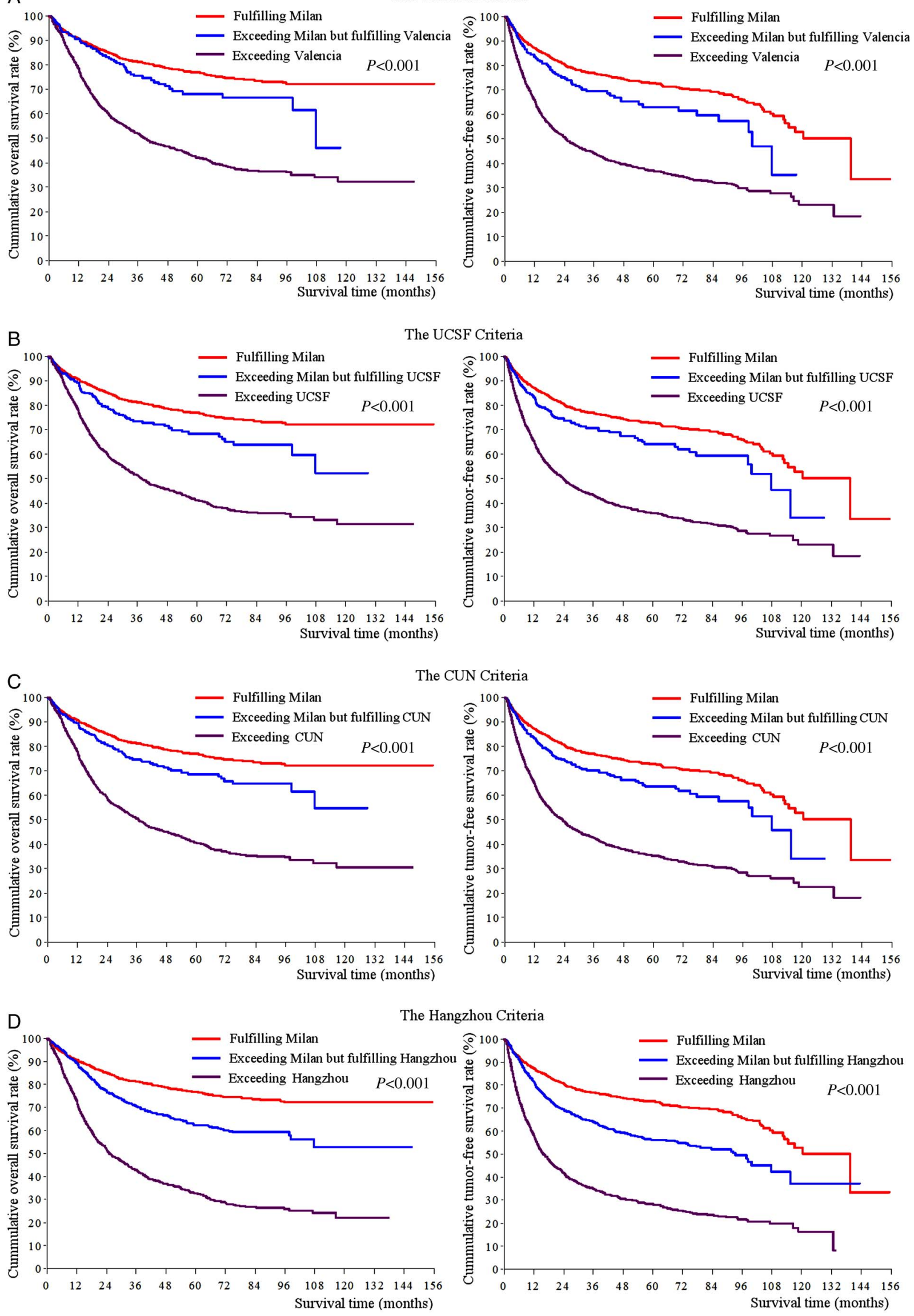

Figure 2 Survival curves for different criteria ( $N=6012)$. The overall and tumour-free survival curves for $(A)$ the Valencia criteria, (B) University of California, San Francisco (UCSF) criteria, (C) University Clinic of Navarra (CUN) criteria and (D) Hangzhou criteria. 


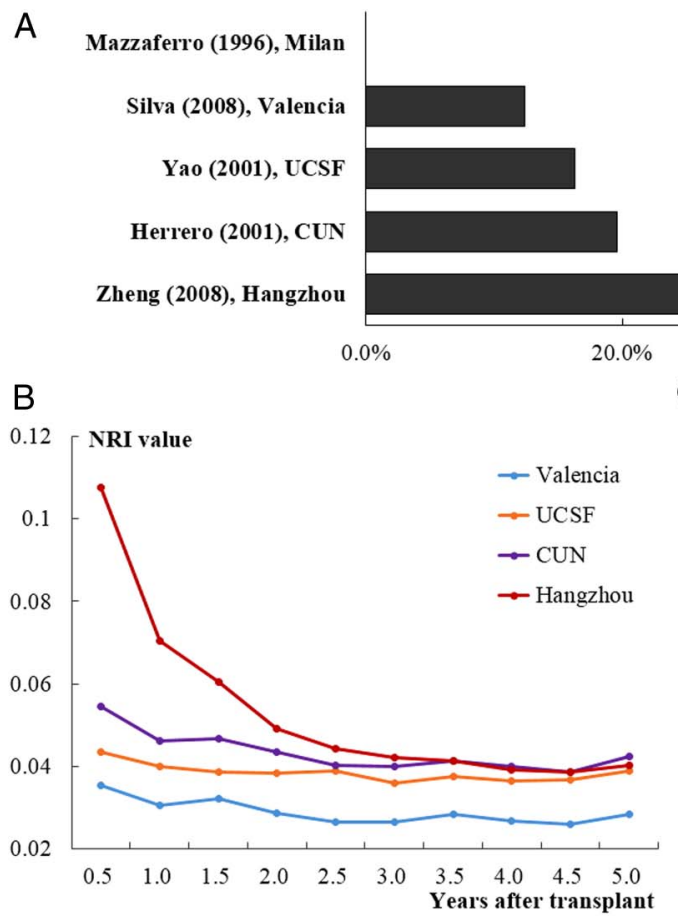

Figure 3 The comparison of different criteria. (A) Increase in the number of eligible hepatocellular carcinoma transplant candidates compared with the Milan criteria ( $\mathrm{N}=6012)$; (B) The time-dependent net reclassification improvement (NRI) curves for different criteria in reference to post-transplant recurrence. Patients censored before the endpoints for analysis were excluded. (C) The time-dependent area under the receiver operating characteristic curve (AUROC) value for different criteria according to death or tumour recurrence. Patients censored before the endpoints for analysis were excluded. CUN, University Clinic of Navarra criteria; UCSF, University of California, San Francisco criteria.

Promisingly, recent studies have defined subsets of patients exceeding the Milan criteria but still with equivalent outcomes. Four well-known expanded criteria derived from these studies were included in this study. As shown in figure $3 \mathrm{~A}$, the different expanded criteria provided an extremely wide variety of increased numbers of eligible candidates, up to one half by the Hangzhou criteria. On the other hand, the overall and tumourfree survivals of patients fulfilling the Valencia, UCSF, CUN and Hangzhou criteria were comparable to those of the Milan criteria (see online supplementary table S2). Volk et al's study ${ }^{21}$ demonstrated that a threshold of $61 \%$ at 5 -year overall survival was demanded to assess the validity of expansion to the Milan criteria, at least in the USA. In our study, the 5-year overall survival rate was $62 \%$ or higher for the newly recruited subsets by

Table 1 Performance of different criteria in the multivariate Cox regression model

\begin{tabular}{|c|c|c|c|c|}
\hline & $\begin{array}{l}\text { LRT } \chi^{2} \\
\text { ( } p \text { value) }\end{array}$ & $\begin{array}{l}\text { Loss } \\
\text { in } \chi^{2}\end{array}$ & AIC & $\begin{array}{l}\text { Changes } \\
\text { in AIC }\end{array}$ \\
\hline Full model & $765.4(<0.001)$ & - & 33413.2 & - \\
\hline Removing Milan & $759.9(<0.001)$ & -5.5 & 33416.6 & 3.4 \\
\hline Removing Valencia & $765.4(<0.001)$ & 5.5 & 33411.2 & -5.4 \\
\hline Removing UCSF & $764.7(<0.001)$ & -0.7 & 33411.8 & 0.6 \\
\hline Removing CUN & $761.3(<0.001)$ & -3.4 & 33415.3 & 3.5 \\
\hline $\begin{array}{l}\text { Removing } \\
\text { Hangzhou }\end{array}$ & $303.6(<0.001)$ & -154.7 & 33570.0 & 154.7 \\
\hline \multicolumn{5}{|c|}{$\begin{array}{l}\text { A multivariate Cox model was built comprising all five sets of criteria as covariates. } \\
\text { By reducing a certain set of criteria individually from the whole model, its } \\
\text { independent contribution was evaluated in regards to the changes in LRT } \chi^{2} \text { and AIC } \\
\text { value. } \\
\text { AIC, Akaike information criterion; CUN, University Clinic of Navarra; LRT, likelihood } \\
\text { ratio test; UCSF, University of California, San Francisco. }\end{array}$} \\
\hline
\end{tabular}

the expanded criteria. The results indicated that the Milan criteria can be expanded.

Although the post-transplant survival is acceptable for the expanded criteria, we still observed decrease in the survival rates for the patients exceeding Milan but fulfilling the expanded criteria compared with those fulfilling Milan. It is a different matter whether those newly recruited patients by the expanded criteria are still good enough to be considered for liver transplant. For our part, a tumour-free survival of $>80 \%$ and $>55 \%$ at 1 and 5 years (in the expansion to the Milan criteria), respectively, is acceptable. Therefore, the patients exceeding Milan but fulfilling the expanded criteria may still be appropriate for liver transplant, particularly in China, which bears the greatest HCC burden worldwide.

Table 2 Risk factors for tumour recurrence in patients exceeding the Milan criteria by multivariate Cox regression $(\mathrm{N}=3386)$

\begin{tabular}{llrllll}
\hline Variables & Group & N & B & $\begin{array}{l}\text { Relative } \\
\text { risk }\end{array}$ & $95 \%$ Cl & p Value \\
\hline Age (years) & $>50$ & 1704 & -0.25 & 0.78 & 0.65 to 0.93 & 0.004 \\
& $\leq 50^{*}$ & 1682 & & & & \\
Cirrhosis & Negative & 509 & 0.10 & 1.11 & 0.92 to 1.35 & 0.30 \\
& Positive* & 2877 & & & & \\
Year of & $<2005$ & 447 & 0.36 & 1.47 & 1.08 to 2.04 & 0.07 \\
transplant & $2005-2010$ & 2214 & 0.19 & 1.21 & 0.97 to 1.87 & \\
& $>2010^{*}$ & 725 & & & & \\
Hangzhou & Fulfilling & 1352 & -0.67 & 0.51 & 0.43 to 0.60 & $<0.001$ \\
criteria & Exceeding & 2034 & & & & \\
\hline $\begin{array}{c}\text { * Reference group. } \\
\text { HBsAg, hepatitis B surface antigen. }\end{array}$
\end{tabular}




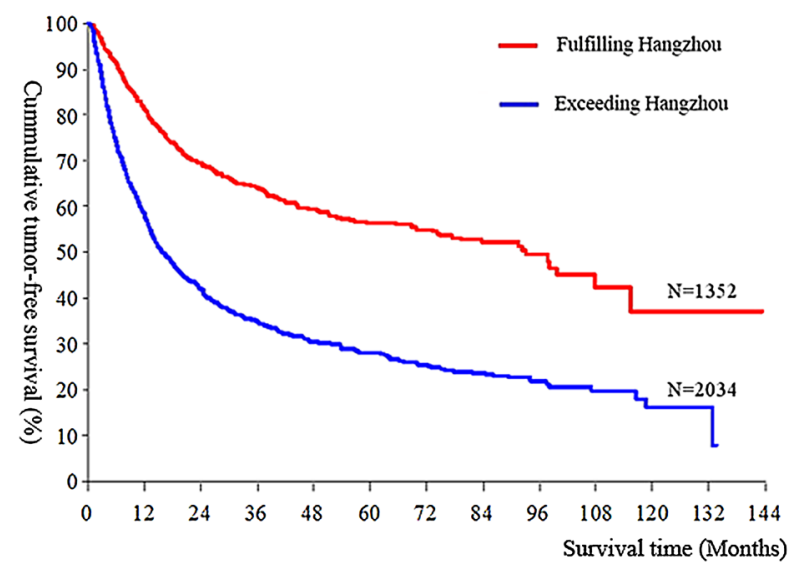

Figure 4 Tumour-free survival curves for patients exceeding the Milan criteria. In patients exceeding the Milan criteria, those fulfilling the Hangzhou criteria had significantly improved tumour-free survival compared with those exceeding it $(p<0.001)$.

Table 3 Risk factors for tumour recurrence in patients exceeding the Milan criteria but fulfilling the Hangzhou criteria by multivariate Cox regression $(\mathrm{N}=1352)$

\begin{tabular}{llllllll}
\hline Variables & Group & N & B & $\begin{array}{l}\text { Relative } \\
\text { risk }\end{array}$ & $95 \%$ Cl & p Value \\
\hline Tumour burden & $5-8$ & 977 & -0.62 & 0.54 & 0.38 to 0.76 & $<0.001$ \\
$(\mathrm{~cm})$ & $>8^{*}$ & 375 & & & & & \\
AFP (ng/mL) & $\leq 100$ & 781 & -1.11 & 0.33 & 0.23 to 0.48 & $<0.001$ \\
& $100-400$ & 280 & -0.61 & 0.54 & 0.36 to 0.82 & \\
& $>400^{*}$ & 291 & & & & & \\
Year of & $<2005$ & 181 & 0.68 & 1.99 & 0.83 to 4.77 & 0.11 \\
transplant & $2005-2010$ & 849 & 0.66 & 1.91 & 1.09 to 3.51 & \\
& $>2010$ & 322 & & & & & \\
\hline *Reference group. & & & & & \\
AFP, $\alpha$-fetoprotein.
\end{tabular}

To provide more evidence to support the expanded criteria, this study then employed the method of NRI analysis, which was proposed by Pencina et al in 2008. ${ }^{14}$ Focusing on the patients exceeding the Milan but fulfilling the expanded criteria, NRI reflects the general changes in the prognostic-classificating efficiency when switching from the Milan criteria to the other. This novel statistical method has presently been applied in survival analysis for medical research. ${ }^{22} 23$ As shown in online supplementary table $\mathrm{S} 4$, the expanded criteria significantly improved the risk reclassification compared with the Milan criteria, indicating that performing transplants on patients exceeding the Milan but fulfilling the expanded criteria brought benefits to the general outcome. Furthermore, if we take the Hangzhou criteria for an instance, 'exceeding the Hangzhou criteria' (considered as a variable) was the independent prognostic factor for tumour recurrence in patients exceeding the Milan criteria. It implied that patients exceeding Milan but fulfilling Hangzhou criteria could achieve a relatively better prognosis. In addition, according to the time-dependent NRI and AUROC curves, the Hangzhou criteria had a distinguished prognostic value in the early years after transplants ( $<2$ years) compared with the other criteria. Meanwhile, now we are trying to improve the long-term posttransplant survival using various ways such as adjuvant chemotherapy, immunotherapy and molecular targeted therapy (sorafenib). Anti-HBV therapy is also of vital importance for the prevention of tumour recurrence. However, considering the shortage of organ sources, more evidence is needed for the choice of selecting criteria in clinical practice.

In patients exceeding the Milan criteria but fulfilling the Hangzhou criteria, we further stratified subgroups to help select the optimal candidates. We found that AFP $\leq 100 \mathrm{ng} / \mathrm{mL}$ and tumour burden $\leq 8 \mathrm{~cm}$ were the only two independent prognostic factors, and the AFP value $\leq 100 \mathrm{ng} / \mathrm{mL}$ was of great value in discriminating those with promising outcomes (table 3 and online supplemental table S6). As shown in figure 5B and online supplementary table S7, the Hangzhou criteria were subsequently stratified as type A (tumour burden $\leq 8 \mathrm{~cm}$, or tumour burden $>8 \mathrm{~cm}$ but with AFP $\leq 100 \mathrm{ng} / \mathrm{mL}$ and well-moderate differentiation) and B (tumour burden $>8 \mathrm{~cm}$ but AFP between 100 and $400 \mathrm{ng} / \mathrm{mL}$ and well-moderate differentiation). In regards to the post-transplant survival of patients in the different types, it is reliable to select type A as the optimal candidate for transplantation. As for the patients in type B, whether neoadjuvant and post-transplant adjuvant therapy would help them achieve acceptable outcomes needs further investigation. On the other hand, considering the relatively poor prognosis as well as the shortage of organ source, Hangzhou B could be regarded as a relative contraindication for liver transplantation.
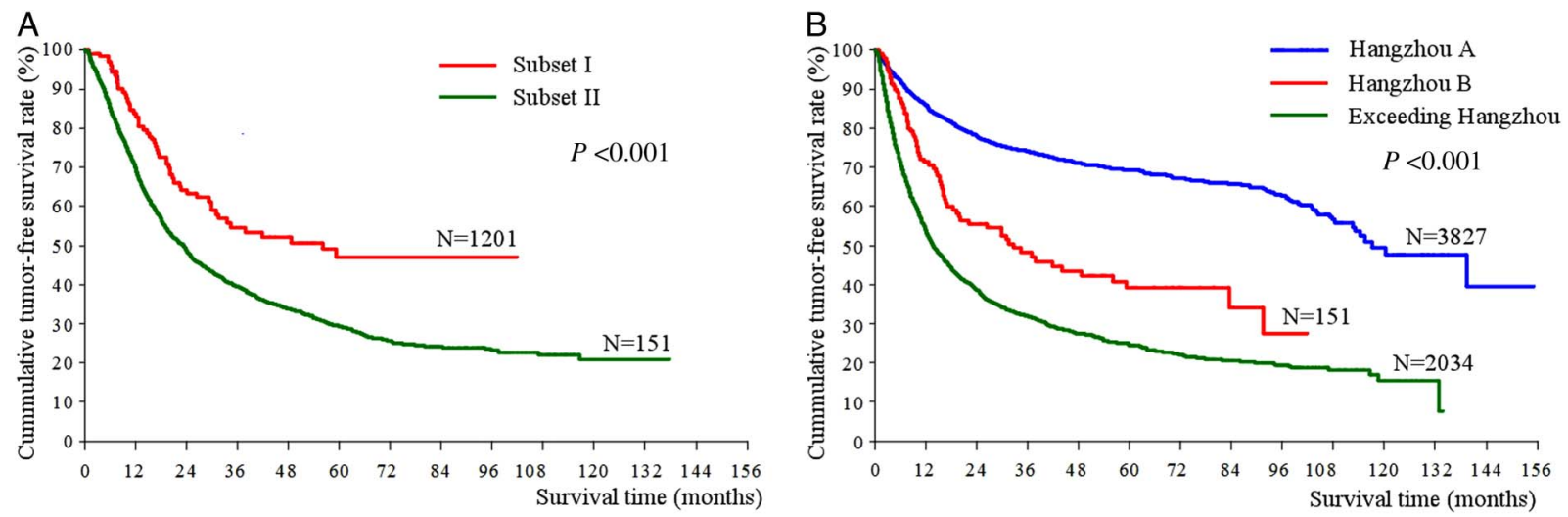

Figure 5 Survival analysis of the subgroup study based on the Hangzhou criteria. (A) Tumour-free survival curves of different subsets of patients exceeding the Milan but fulfilling Hangzhou criteria. Subset I: tumour burden $\leq 8 \mathrm{~cm}$ or $\alpha$-fetoprotein (AFP) $\leq 100 \mathrm{ng} / \mathrm{mL}$; subset II: tumour burden $>8 \mathrm{~cm}$ but AFP between 100 and $400 \mathrm{ng} / \mathrm{mL}$. Subset I had significantly better prognosis than Subset II $(p<0.001)$. (B) The tumour-free survival curves for the stratified Hangzhou criteria. The Hangzhou criteria were stratified into (1) type A: tumour burden $\leq 8 \mathrm{~cm}$ or AFP $\leq 100 \mathrm{ng} / \mathrm{mL}$; $(2)$ type B: tumour burden $>8 \mathrm{~cm}$ but AFP between 100 and $400 \mathrm{ng} / \mathrm{mL}$. Type A had significantly better prognosis than type B $(p<0.001)$. Both types $A$ and $B$ had significantly improved prognosis compared with those patients exceeding the Hangzhou criteria $(p<0.001)$. 
A limitation of this study was that both the establishment and verification of the Hangzhou criteria were based on the Chinese population. It should also be clarified whether the Hangzhou criteria are effective and safe in Western cohorts. Another issue to consider is the need of pretransplant differentiation grading in the Hangzhou criteria. In fact, the Hangzhou criteria suggest a need of biopsy only in the situation of tumour burden $>8 \mathrm{~cm}$ and AFP $\leq 400 \mathrm{ng} / \mathrm{mL}(920 / 6012$ of the whole cohort) since tumour burden and AFP should be adequate for the judgements in all the other cases. Moreover, biopsy can be performed safely with experienced doctors and standard procedures. ${ }^{24} 25$ Also, there exist other valuable criteria that were not included in this study, such as the Up-to-7 criteria, ${ }^{26}$ that require information about microvascular invasion.

In conclusion, the Milan criteria can be safely and effectively expanded. The prognostic stratification system based on the Hangzhou criteria serves as a hierarchy of transplant candidates for HCC. Patients fulfilling Hangzhou type A should have the priority for liver transplantation.

Acknowledgements We gratefully acknowledge Prof. Sheung-Tat Fan from the Department of Surgery, Queen Mary Hospital, the University of Hong Kong, and Prof. Ronald W. Busuttil from the Dumont-UCLA Transplant Center, David Geffen School of Medicine at UCLA, Department of Surgery, Los Angeles, USA, for reviewing this manuscript, and the China Liver Transplant Registry (CLTR) for the data collection and statistical analysis. We also acknowledge the transplant centres from Mainland China for their major contribution to the CLTR database.

Contributors Study concept: SZ. Study design: SZ, XX, JW, LZ, SY, LW, LG, QL and DL. Interpretation analysis: XX, DL, QL, XW, ZT and HX. Acquisition of data: HW and WJ. Manuscript drafting: DL, XX, QL, XW, QK and FG. Revising the manuscript: $D L, X X, W W, M Z$ and YS. Manuscript final version approval: All authors.

Funding This work was supported by the National High-tech R\&D Program of China (863 Program) (no. 2012AA020204), National S\&T Major Project (no. 2012ZX10002017) and the Cheung Kong Scholars Program of China.

\section{Competing interests None.}

Patient consent Obtained.

Ethics approval Institutional Review Board (2014, no. 293), the First Affiliated Hospital, Zhejiang University School of Medicine,. CLTR started the research after obtaining the approval of Ethics Committee from each participating centre according to the Regulations on Human Organ Transplant and national legal requirements. The research design was hospital-based and retrospective. The research was approved by the CLTR (http://www.cltr.org/), which was authorised as the only national liver transplantation registry in Mainland China by the Ministry of Health in May 2008. The data warehouse is administered by the Center of Study for Liver Disease, Department of Surgery, Queen Mary Hospital, University of Hong Kong.

Provenance and peer review Not commissioned; externally peer reviewed.

Data sharing statement This article was published as an abstract in 'The ILTS 20th Annual International Congress', Liver Transplantation, 2014.

Open Access This is an Open Access article distributed in accordance with the Creative Commons Attribution Non Commercial (CC BY-NC 4.0) license, which permits others to distribute, remix, adapt, build upon this work non-commercially, and license their derivative works on different terms, provided the original work is properly cited and the use is non-commercial. See: http://creativecommons.org/ licenses/by-nc/4.0/

\section{REFERENCES}

1 Venook AP, Papandreou C, Furuse J, et al. The incidence and epidemiology of hepatocellular carcinoma: a global and regional perspective. Oncologist 2010;15 (Suppl 4):5-13.
2 Mazzaferro V, Bhoori S, Sposito C, et al. Milan criteria in liver transplantation for hepatocellular carcinoma: an evidence-based analysis of 15 years of experience. Liver Transp/ 2011;17(Suppl 2):S44-57.

3 Shi J, Zhu L, Liu S, et al. A meta-analysis of case-control studies on the combined effect of hepatitis $B$ and $C$ virus infections in causing hepatocellular carcinoma in China. Br J Cancer 2005:92:607-12.

4 Parkin DM, Bray F, Ferlay J, et al. Global cancer statistics, 2002. CA Cancer J Clin 2005;55:74-108

5 Yokoyama I, Todo S, Iwatsuki S, et al. Liver transplantation in the treatment of primary liver cancer. Hepatogastroenterology 1990;37:188-93.

6 Mazzaferro V, Regalia E, Doci R, et al. Liver transplantation for the treatment of small hepatocellular carcinomas in patients with cirrhosis. N Engl J Med 1996:334:693-9.

7 Yao FY. Expanded criteria for liver transplantation in patients with hepatocellular carcinoma. Hepatol Res 2007;37(Suppl 2):S267-74.

8 Yao FY, Ferrell L, Bass NM, et al. Liver transplantation for hepatocellular carcinoma: expansion of the tumor size limits does not adversely impact survival. Hepatology 2001;33:1394-403.

9 Herrero Jl, Sangro B, Quiroga J, et al. Influence of tumor characteristics on the outcome of liver transplantation among patients with liver cirrhosis and hepatocellular carcinoma. Liver Transp/ 2001;7:631-6.

10 Silva M, Moya A, Berenguer M, et al. Expanded criteria for liver transplantation in patients with cirrhosis and hepatocellular carcinoma. Liver Transp/ 2008;14:1449-60.

11 Zheng SS, Xu X, Wu J, et al. Liver transplantation for hepatocellular carcinoma: Hangzhou experiences. Transplantation 2008;85:1726-32.

12 Prasad KR, Young RS, Burra P, et al. Summary of candidate selection and expanded criteria for liver transplantation for hepatocellular carcinoma: a review and consensus statement. Liver Transp/ 2011;17(Suppl 2):S81-9.

13 Petrou A, Xynos ID, Tsigritis K, et al. The significance of DNA image cytometry and Edmondson-Steiner grading on prognosis after curative resection of hepatocellular carcinoma. J BUON 2011;16:93-7.

14 Pencina MJ, D'Agostino RS, D'Agostino RJ, et al. Evaluating the added predictive ability of a new marker: from area under the ROC curve to reclassification and beyond. Stat Med 2008;27:157-72, 207-12.

15 Bandos Al, Rockette HE, Song T, et al. Area under the free-response ROC curve (FROC) and a related summary index. Biometrics 2009;65:247-56.

16 Marrero JA, Fontana RJ, Barrat A, et al. Prognosis of hepatocellular carcinoma: comparison of 7 staging systems in an American cohort. Hepatology 2005:41:707-16.

17 Cillo U, Vitale A, Grigoletto F, et al. Prospective validation of the Barcelona Clinic Liver Cancer staging system. J Hepatol 2006;44:723-31.

18 Ueno S, Tanabe G, Sako K, et al. Discrimination value of the new western prognostic system (CLIP score) for hepatocellular carcinoma in 662 Japanese patients. Cancer of the Liver Italian Program. Hepatology 2001;34:529-34.

19 Chaurasia A, Harel 0. Using AIC in Multiple Linear Regression framework with Multiply Imputed Data. Health Serv Outcomes Res Methodol 2012; 12:219-33.

20 Sharma P, Balan V, Hernandez JL, et al. Liver transplantation for hepatocellular carcinoma: the MELD impact. Liver Transp/ 2004;10:36-41.

21 Volk ML, Vijan S, Marrero JA. A novel model measuring the harm of transplanting hepatocellular carcinoma exceeding Milan criteria. Am J Transplant 2008;8:839-46

22 Zheng Y, Parast L, Cai T, et al. Evaluating incremental values from new predictors with net reclassification improvement in survival analysis. Lifetime Data Anal 2013;19:350-70

23 Duvoux C, Roudot-Thoraval F, Decaens T, et al. Liver transplantation for hepatocellular carcinoma: a model including alpha-fetoprotein improves the performance of Milan criteria. Gastroenterology 2012:143:986-94, e14-5.

24 Colecchia A, Scaioli E, Montrone L, et al. Pre-operative liver biopsy in cirrhotic patients with early hepatocellular carcinoma represents a safe and accurate diagnostic tool for tumour grading assessment. J Hepatol 2011;54:300-5.

25 Ozkara SK, Tuneli IO. Fine Needle Aspiration Cytopathology of Liver Masses: 101 Cases with Cyto-/Histopathological Analysis. Acta Cytol 2013;57:332-6.

26 Mazzaferro V, Llovet JM, Miceli R, et al. Predicting survival after liver transplantation in patients with hepatocellular carcinoma beyond the Milan criteria: a retrospective, exploratory analysis. Lancet Oncol 2009;10:35-43. 